\title{
Research Paper \\ The Role of Stroop Performance in Predicting Sleep Quality and Quality of Life in the Elderly
}

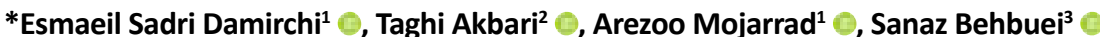

1. Department of Counseling, Faculty of Educational Sciences and Psychology, University of Mohaghegh Ardabili, Ardabil, Iran.

2. Department of Educational Sciences, Faculty of Educational Sciences and Psychology, University of Mohaghegh Ardabili, Ardabil, Iran.

3. Department of Psychology, Faculty of Education and Psychology, University of Tabriz, Tabriz, Iran.

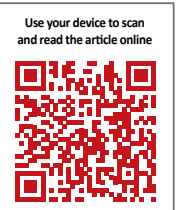

CFtation Sadri Damirchi E, Akbari T, Mojarrad A, Behbuei S. [The Role of Stroop Performance in Predicting Sleep Quality and Quality of Life in the Elderly (Persian)]. Salmand: Iranian Journal of Ageing. 2019; 13(Special Issue):564-575. https://doi. org/10.32598/SIJA.13.Special-Issue.564

https://doi.org/10.32598/SIJA.13.Special-Issue.564

Key words:

Attention function, Elderly, Sleep Quality, Quality of Life

\section{A B STRACT}

Received: 19 Jul 2018 Accepted: 28 Dec 2018 Available Online: 10 Mar 2019

Objectives Today, the life expectancy of elderly people has increased all over the world. The purpose of this study was to investigate the role of attention performance in predicting the elderly's sleep quality and quality of life.

Methods \& Materials The research design was descriptive correlational. The statistical population consisted of all elderly men over 60 years of age who live in the nursing homes of Ardabil City, Iran, in 2017. Out of them, a sample of 200 individuals was selected by the available sampling method. Pittsburg sleep quality questionnaire, the World Health Organization quality of life and Stroop questionnaire were used for data collection. The obtained data were analyzed by the Pearson correlation and regression analysis in SPSS-21.

Results The findings showed that the Mean \pm SD of the subjects' age was $66.47 \pm 6.756$ years and their quality of life and sleep quality Mean \pm SD scores were $73.95 \pm 21.41$ and $5.55 \pm 6.36$, respectively. Also, the Mean $\pm S D$ scores of Stroop cards of error, correct, response, and interference were $6.54 \pm 8.52,94.48 \pm 7.37$, $121.56 \pm 61.33$, and $50.44 \pm 58.08$, respectively. The results of the Pearson correlation and regression analysis showed a significant relationship between performance in the Stroop test and the manner of attention with the quality of sleep and quality of life $(P<0.01, \beta=0.351)$, and the way in which attention is given in the Stroop test can predict the quality of sleep $(P<0.001)$ and quality of life $(P<0.001, \beta=0.308)$.

Conclusion The performance of the Stroop test in the elderly by conducting training and performing different therapeutic classes will improve their quality of life and sleep quality.

\section{Extended Abstract}

\section{Objectives}

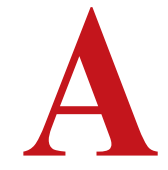

ging is a natural process of life that occurs due to gradual changes in metabolic activities of the organs and the inability of cell regenerative capacity. According to the World Health Organization
(WHO) report, there are currently 600 million elderly people living around the world and their number is expected to be doubled by 2025 and reach 2 billion by 2050 [1]. So WHO has named this century for aging and its slogan for 2012 was "Good health adds life to years" [2, $3]$. Considering the increasing population of the elderly and the many problems that associate with this population, the purpose of this study was to investigate the

\section{* Corresponding Author}

\section{Esmaeil Sadri Damirchi, PhD.}

Address: Department of Counseling, Faculty of Educational Sciences and Psychology, University of Mohaghegh Ardabili, Ardabil, Iran. Tel: +98 (45) 31505644

E-mail: e.sadri@uma.ac.ir 
role of attention performance in predicting the quality of sleep and quality of life in people aged over 60 years.

\section{Methods \& Materials}

This is a descriptive correlational study. The study population consisted of all older adults aged over 60 years living in the elderly nursing homes in Ardabil City, Iran in 2017. Using a convenience sampling method, 200 older people were selected as study samples. For collecting data, the following tools were used:

\section{Stroop effect test}

This test was introduced by Stroop in 1935 for measuring selective attention and cognitive flexibility. Since then, different types of test have been presented. The reliability of this test for the first and second cards is 0.88 and for the third and fourth cards is 0.80 [4].

\section{Pittsburgh Sleep Quality Index (PSQI)}

This questionnaire was designed by Buysse and his colleagues in 1989 and has 18 items scored from 0 to 3 , where score 3 indicates negative state and 0 positive state. For measuring sleep quality, their scores are summed up, where the scores $0-4$ indicate good quality and score 5 or greater shows poor quality [5]. PSQI has an internal consistency coefficient of about 0.80 , and the test-retest reliability of its Persian version is between 0.93 and 0.98 reported by Pakpour et al. $[6,7]$.

\section{The World Health Organization Quality of Life (WHOQOL)-BREF}

This questionnaire has 26 items rated based on 5-point Likert-type type scale. The score of each domain ranged from 0 to 100 ; higher scores show a better quality of life. Reliability of its Persian version for normal people in Teh- ran was reported more than 0.70 which proves the repeatability of this test. It also has a Cronbach $\alpha$ coefficient of more than 0.70 . The validity of this questionnaire is also acceptable in all domains [8].

The collected data were analyzed using regression analysis and the Pearson correlation test, where the significance level was set at $0.05(\mathrm{P}<0.05)$.

\section{Results}

The Mean \pm SD age of the participants (married) was $66.47 \pm 6.576$ years. Table 1 presents descriptive statistics for the variables of Sleep Quality (SQ), Quality of Life (QOL), and Stroop effect test components. As seen, the Mean \pm SD QOL score was $73.95 \pm 21.41$, and the Mean \pm SD SQ score was 5.55 \pm 6.36 . After performing Stroop task, the obtained Mean \pm SD scores of error, correct answer, reaction, and interference were $6.54 \pm 8.52,94.48 \pm 7.37$, $121.56 \pm 61.33$, and $50.44 \pm 58.08$, respectively.

The Pearson correlation test was carried out to examine the relationship between QOL and SQ with Stroop performance. Before that, the normality of data distribution was confirmed by the Kolmogorov-Smirnov test. Table 2 presents the results of the Pearson correlation test. According to the results, there is a significant relationship between error, correction, and interference in the Stroop test and QOL, while SQ had a significant correlation with error and correction in the Stroop test. Hence, with the increase of attention in performing Stroop test, sleep quality and quality of life of older adults can be improved and vice versa.

For examining the role of Stroop performance in predicting QOL and SQ among subjects, regression analysis was used (Table 3). The coefficient of determination in the regression model for OOL and SQ were 0.390 and 0.690, respectively. Based on beta coefficient values, one unit in-

Table 1. The mean and standard deviation of study variables

\begin{tabular}{ccc}
\hline Components & Mean & SD \\
\hline QOL & 73.95 & 21.41 \\
\hline SQ & 5.55 & 6.36 \\
\hline Error (Stroop task) & 6.54 & 8.52 \\
\hline Correct (Stroop task) & 94.48 & 7.37 \\
\hline Reaction time (Stroop task) & 121.56 & 61.33 \\
\hline Interference (Stroop task) & 50.44 & 58.08 \\
\hline
\end{tabular}

QoL: Quality of Life, SQ: Sleep Quality. 
Table 2. The Pearson correlation test results for comparing QOL and SQ with Stroop performance

\begin{tabular}{ccc}
\hline Stroop Performance & QOL & SQ \\
\hline Error & $-0.868^{*}$ & $0.946^{*}$ \\
\hline Correct & $0.568^{*}$ & $-0.595^{*}$ \\
\hline Reaction & -0.110 & 0.044 \\
\hline Interference & $-0.223^{*}$ & 0.083 \\
\hline
\end{tabular}

QoL: Quality of Life, SQ: Sleep Quality

ARGEINING

$* \mathrm{P}<0.01$

Table 3. Regression analysis results for predicting QOL and SQ based on Stroop performance

\begin{tabular}{cccccccc}
\hline Dependent Variable & Independent Variables & $\mathbf{B}$ & $\mathbf{S E}$ & $\boldsymbol{\beta}$ & $\mathbf{t}$ & $\mathbf{R}^{\mathbf{2}}$ & $\mathbf{P}$ \\
\hline \multirow{2}{*}{ Stroop performance } & $\mathrm{QOL}$ & 0.106 & 0.031 & 0.308 & 3.38 & 0.390 & 0.001 \\
& $\mathrm{SQ}$ & 0.405 & 0.105 & 0.351 & 3.86 & 0.690 & 0.000 \\
\hline
\end{tabular}

QoL: Quality of Life, SQ: Sleep Quality.

$\stackrel{\text { IRanian ounnal of }}{A} \in \mathbb{N}$

crease in Stroop performance increases QOL and SQ levels $30.8 \%$ and $35.1 \%$, respectively.

\section{Conclusion}

Based on the results, Stroop performance is significantly related to sleep quality. This result is consistent with the results of Tsapanou et al. [9] and Engleman and Douglas [10]. It was also found out that Stroop performance had a relationship with quality of life which is in agreement with the findings of Gathright et al. [11] and Ediebah et al. [12] studies. The Stroop performance was able to predict sleep quality and quality of life of the elders. Therefore, by providing training programs and performing different group therapies, the Stroop performance can be improved in the elderly, thereby indirectly increasing their quality of life and sleep.

\section{Ethical Considerations}

Compliance with ethical guidelines

In order to comply with the research ethics, after selecting study samples, the purpose of study was explained to the participants and they were free to withdraw from participation in the study at any time.
Funding

This research did not receive any specific grant from funding agencies in the public, commercial, or not-forprofit sectors.

\section{Authors' contributions}

Conceptualization: All authors; Methodology: All authors; Software: All authors; Validation: All authors; Formal analysis: All authors; Investigation: All authors; Resources: All authors; Data curation: All authors; Writing-original draft preparation: All authors; Writingreview \& editing: All authors; Visualization: All authors; Supervision: Esmaeil Sadri Damirchi; Project Administration: Esmaeil Sadri Damirchi; Funding acquisition: All authors.

\section{Conflict of interest}

The authors declared no conflict of interest.

\section{Acknowledgments}

We are grateful for the cooperation of the Rayehe Omid Center. 


\title{
نقش عملكرد توجه در بيشبينى كيفيت خواب و كيفيت زندگى در سالمندان
}

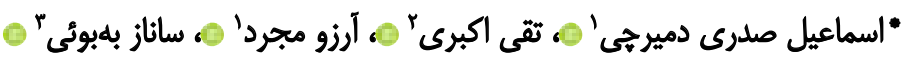 \\ 1- كروه مشاوره، دانشكده علومتربيتى و روانشئاسى، دانشكاه محقق اردييلى اردبيل، ايران.

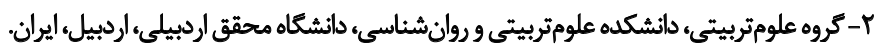

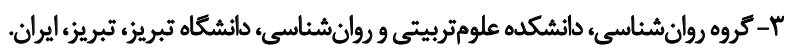

\begin{abstract}
حكبد

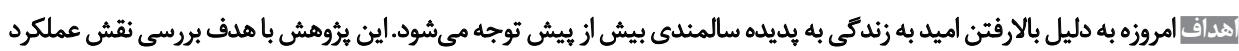

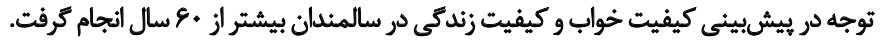

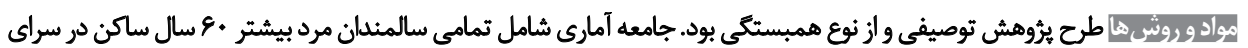

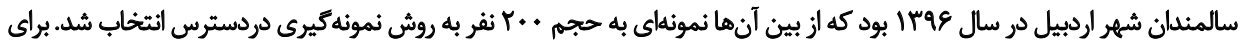

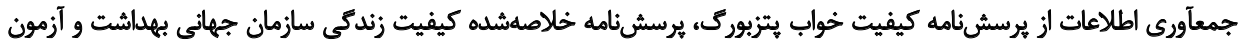

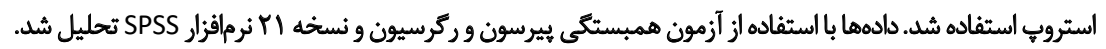

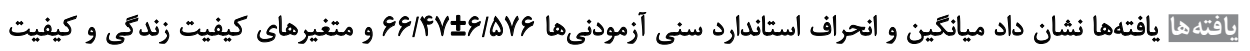

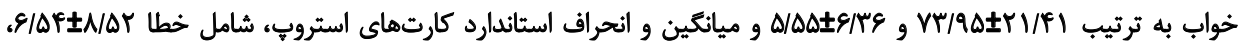

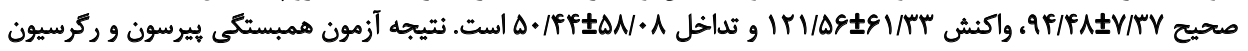

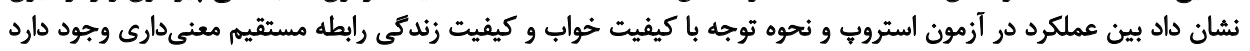

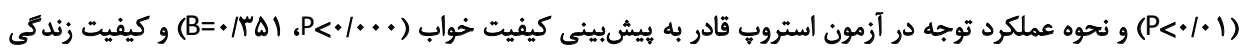

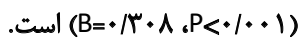

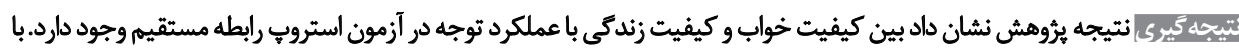

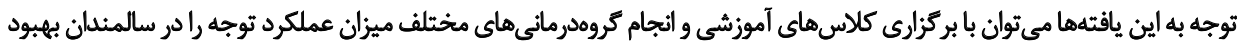

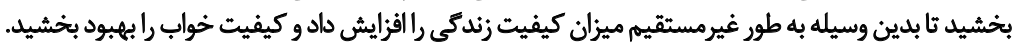

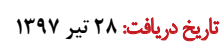

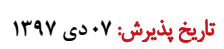

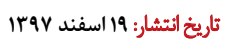

ميليون و بوس هزار نفر خواهد رسيد كه جمعيتى معادل جع درصد از كل جامعه است [r] dales با توجه به افزايش جمعيث اين زروه سنى، سازمان بهداشت

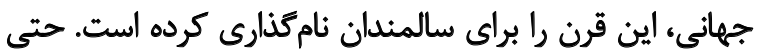

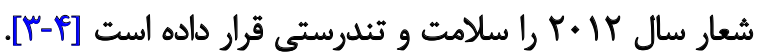

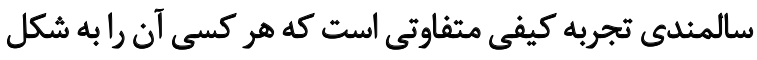

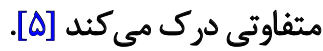

يكى از مشكلات اساسى سالمندان موضوع سوكيرى توجه

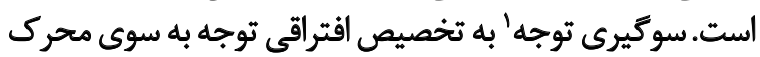

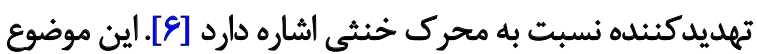

1. Bound attention سالمندى فرايند طبيعى زندكي است كه به دليل تغييرات تدريجى در فعاليتهاى متابوليكى اندامها و ناتوانى ظرفي زئيت

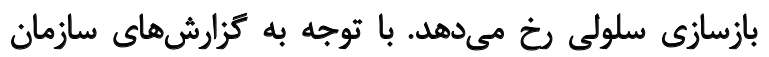

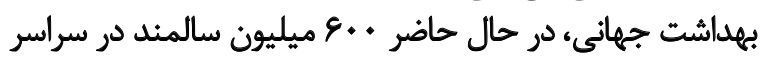

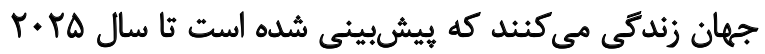

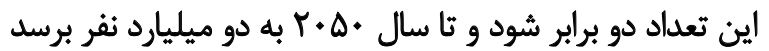

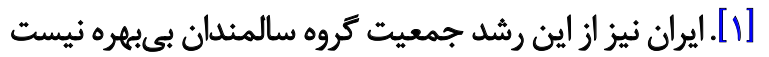

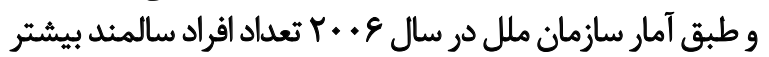

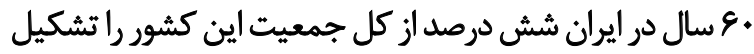

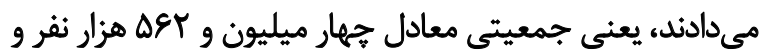

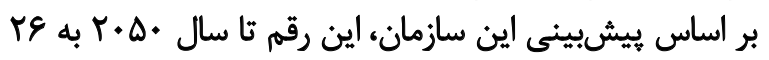

\section{-}


[19] خواب همجنين به رشد و ترميم، يادكيرى و تقويت حافظه

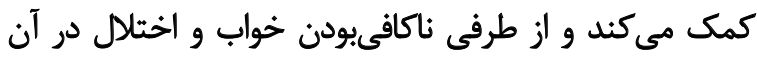

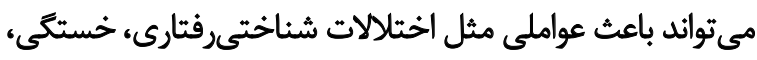

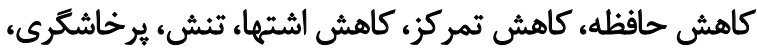

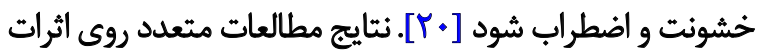

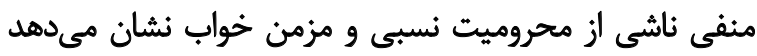

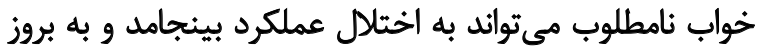

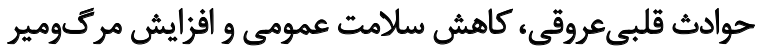

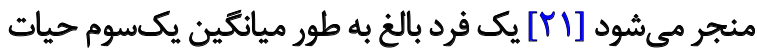

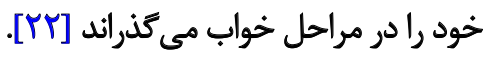
كميت خواب با كيفيت تعامل اجتماعى مرتبط شناخته شده

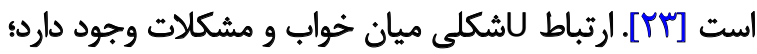

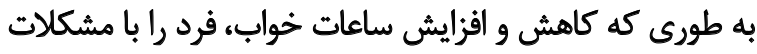

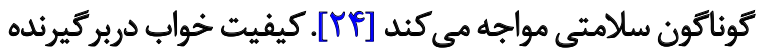

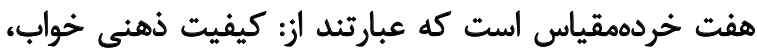

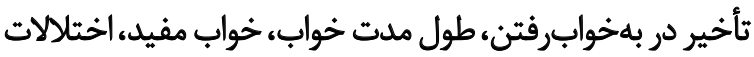

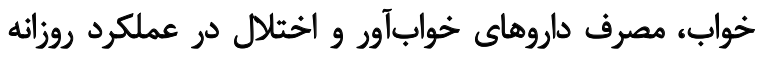

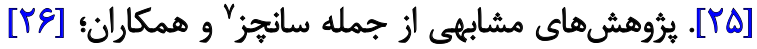

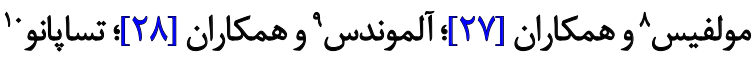

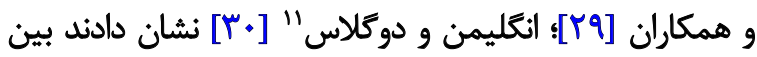

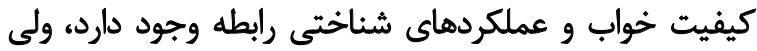

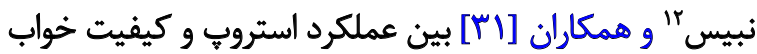
رابطه معنادارى رانيافتند.

با توجه به نبود بيشينه بثروهشى كافى از يك سو و از سوى بإي

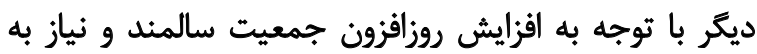

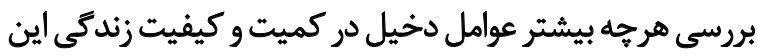

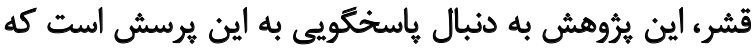

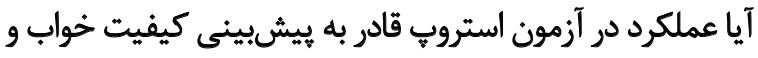

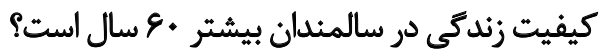

$$
\text { ووشي مطالعه }
$$

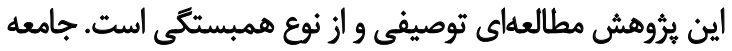

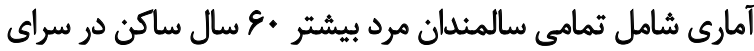

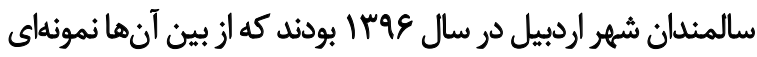

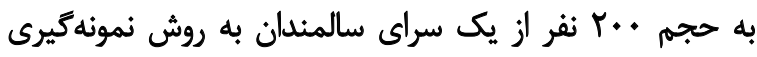

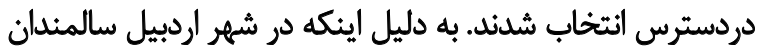

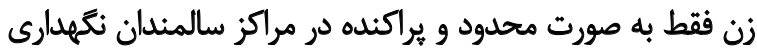

\section{Herrero-Sánchez}

8. Molfese

9. Almondes

10. Tsapanou

11. Engleman

12. Nebes
از دو منظر بررسى شده است. نخست از منظر فرايند و دوم از

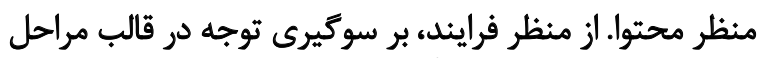

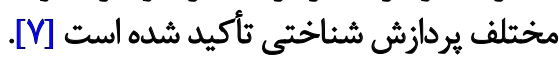
در بحث محتوا، يُؤوهشَّران سعى كرداند محتواى فكرى

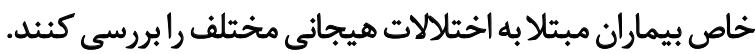

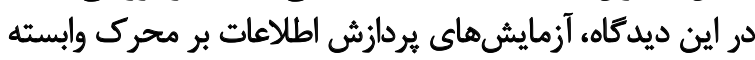

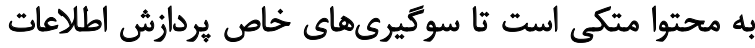

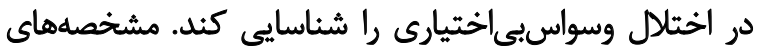

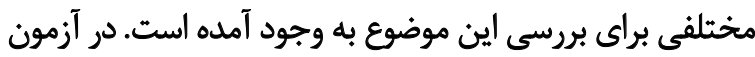

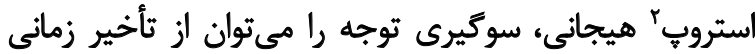

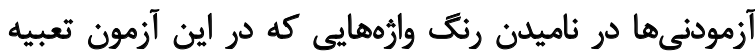

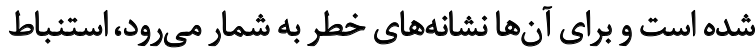

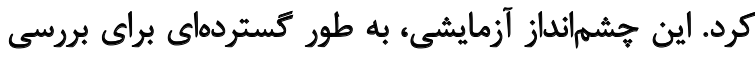

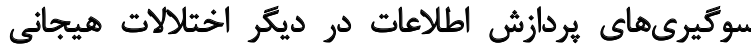
استفاده شده است [1]].

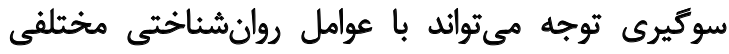

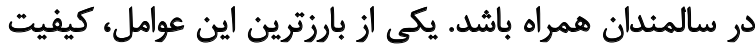

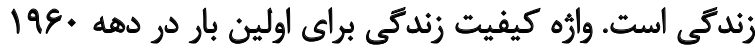

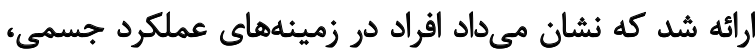

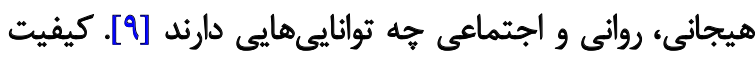

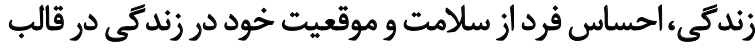

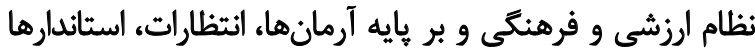

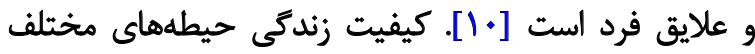

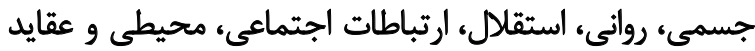

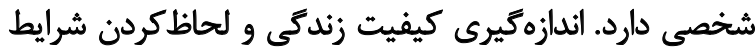

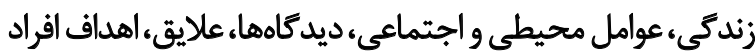

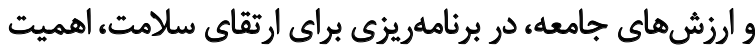

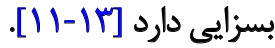

عوامل فيزيولوزيكى، اجتماعى، روانى، اقتصادى، محيطى زئي

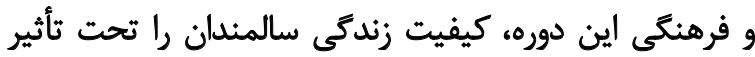

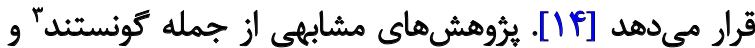

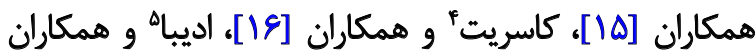

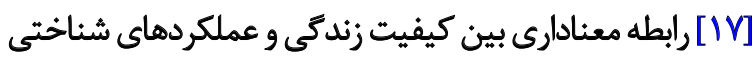

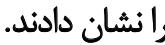

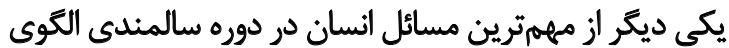

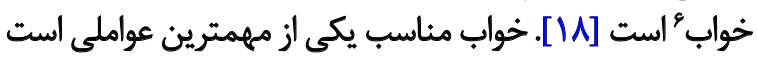

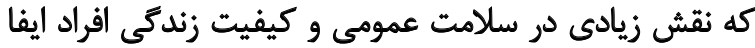
مى كند و موجب بهبود قوادى جسمى دمى روانى و هيجانى مى

2. Stroop Test

3. Gunstad

4. Gathright

5. Ediebah

6. Sleep pattern 
وارهها را بدون توجه به رنك آنها بخواند و در كارت جهارم از راز

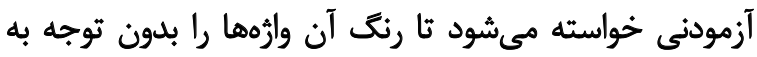

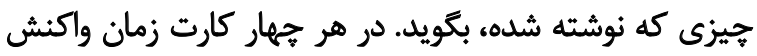

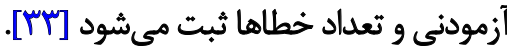

به منظور نمرهدهى و تفسير نتايج حاصل از اين آزمون، نمرات

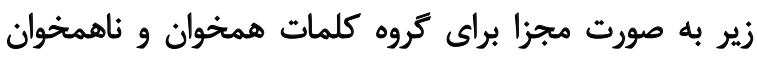

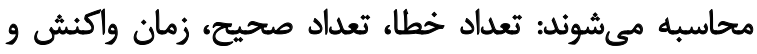

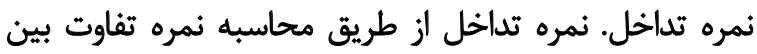

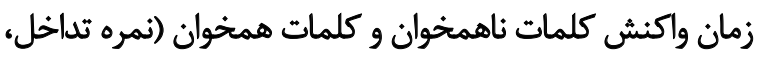

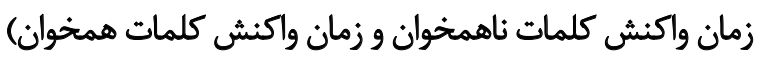

محاسبه مىشود [FF].

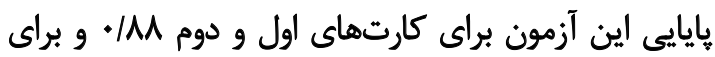

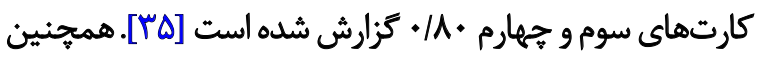

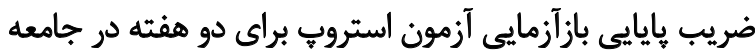

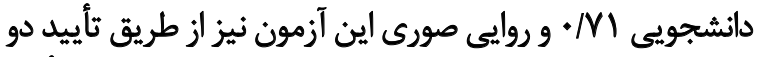

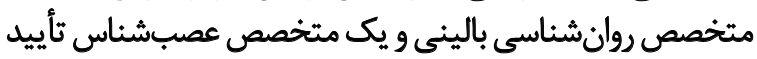

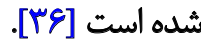

\section{يرسشنامه كيفيت شُواب يتزبورى"}

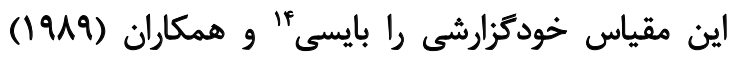

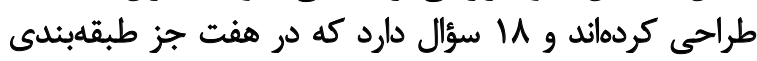

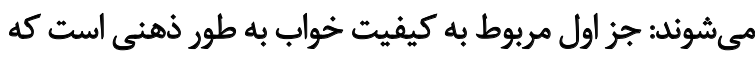

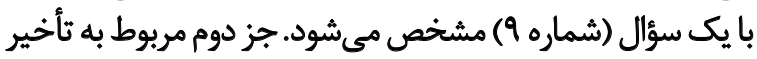

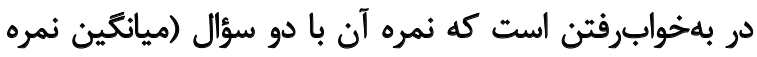

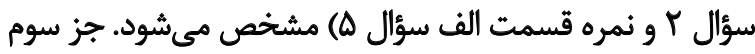

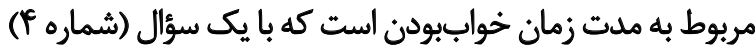

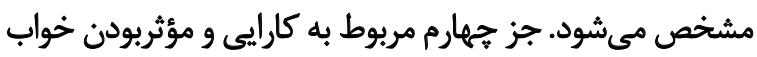

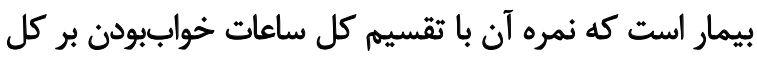

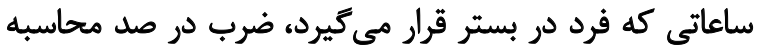

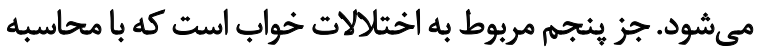

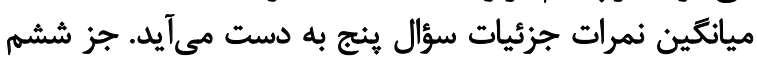
مربوط به مصرف داروهاي خواب آور است كه بـ با يك سؤال (شماره

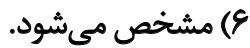

جز هفتم نيز مربوط به عملكرد نامناسب در طول روز است

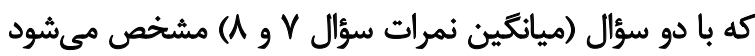

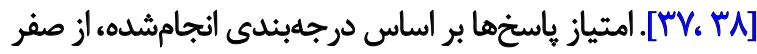

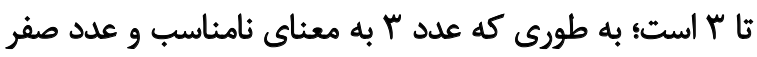

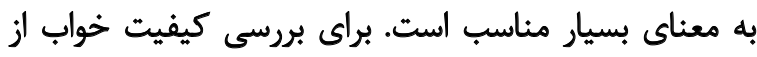

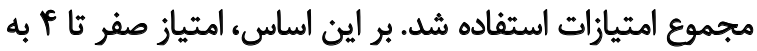

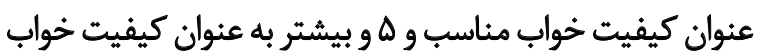

13. Petsburg Sleep Quality Questionnaire (PSQI) 14. Daniel J Buysee
مىشوند براي انسجام بيشتر يرُوهش و دسترسى به نمونهان كان كافى،

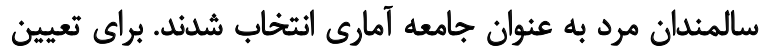

$$
n=\frac{N z^{2} s^{2}}{N d^{2}+z^{2} s^{2}}
$$

حجم نمونه از فرمول زير استفاده شد [بسان]:

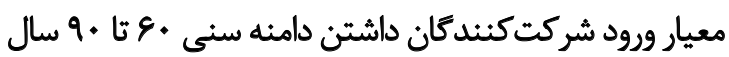

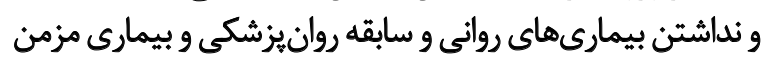

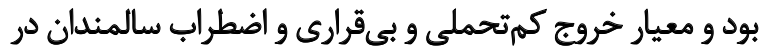

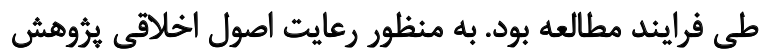

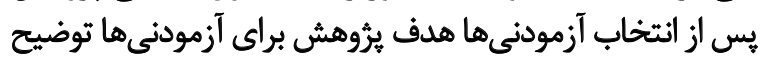

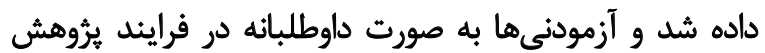

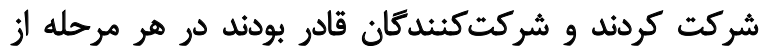

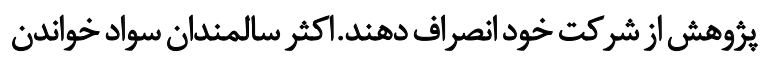

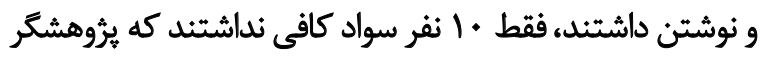

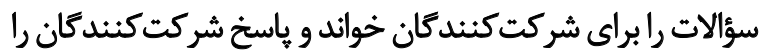
يادداشت كرد. براى تجزيه و تحليل اطلاعات از آز آزمون همبستئى

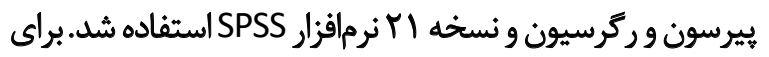
جمعآورى اطلاعات از ابزارهاى زير استفاده شد.

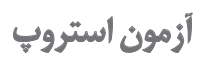

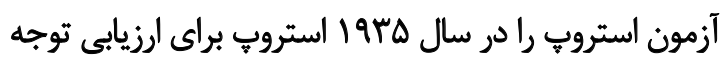

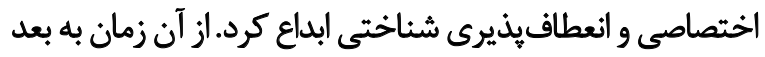

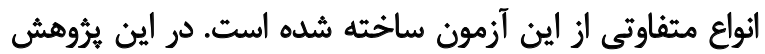

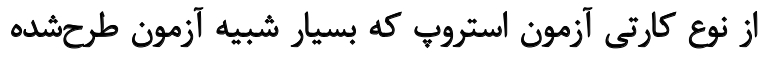

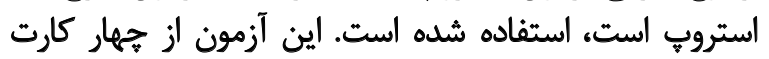

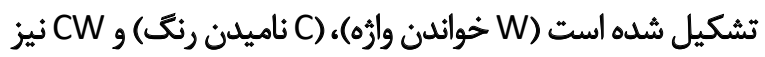

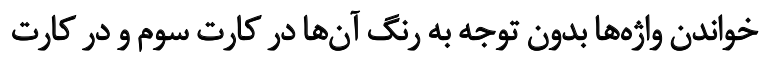

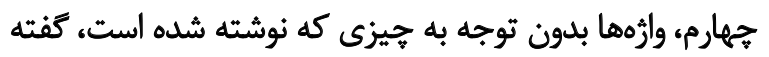

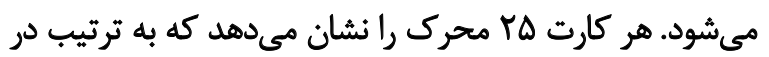
ينج سطر و ينج ستون تنظيم شدان ماند

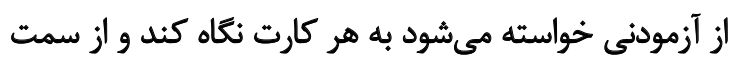

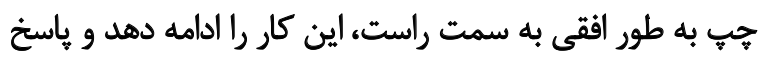

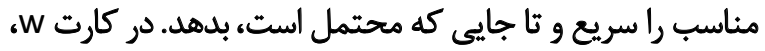

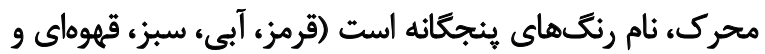

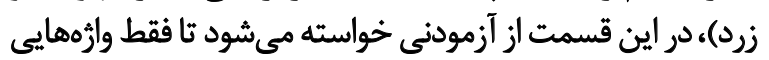

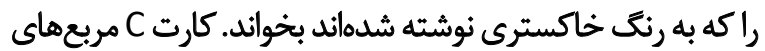

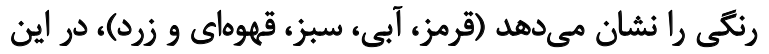

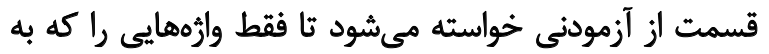

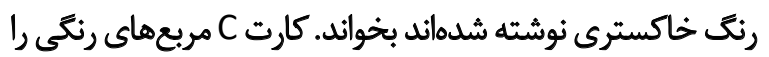

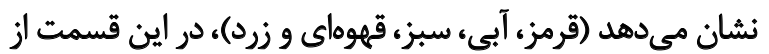

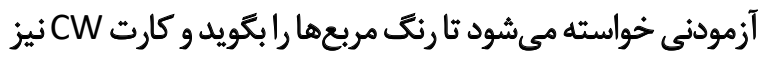

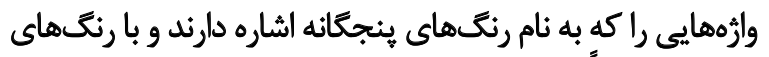

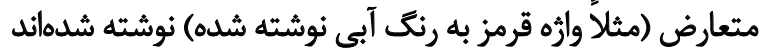

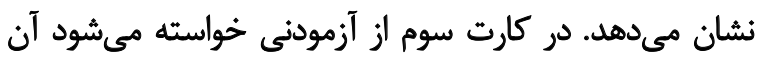


جدول أ أمار مهاى توصيفي متغيرهاي هُوُوهش

\begin{tabular}{|c|c|c|}
\hline انحراف استاندارد & مياتكَين & مؤلفهـا \\
\hline$M / F i$ & $n \pi / 9 \Delta$ & كيفيت زندكى \\
\hline e/re & $\Delta / \Delta \Delta$ & كيفيت خواب \\
\hline NAY & s/DF & خط \\
\hline$V / T V$ & qf/fA & صحen \\
\hline $8 / / H$ & $M T / D E$ & واكنش \\
\hline$\Delta \mathbb{N} \cdot \mathbf{A}$ & $\Delta \cdot / \mu f$ & ت تداخل \\
\hline
\end{tabular}

2

جدول r. نتايج آزمون همبستكى بيرسون براى بررسى كيفيت زئدكى و كيفيت خواب با عملكرد استروب

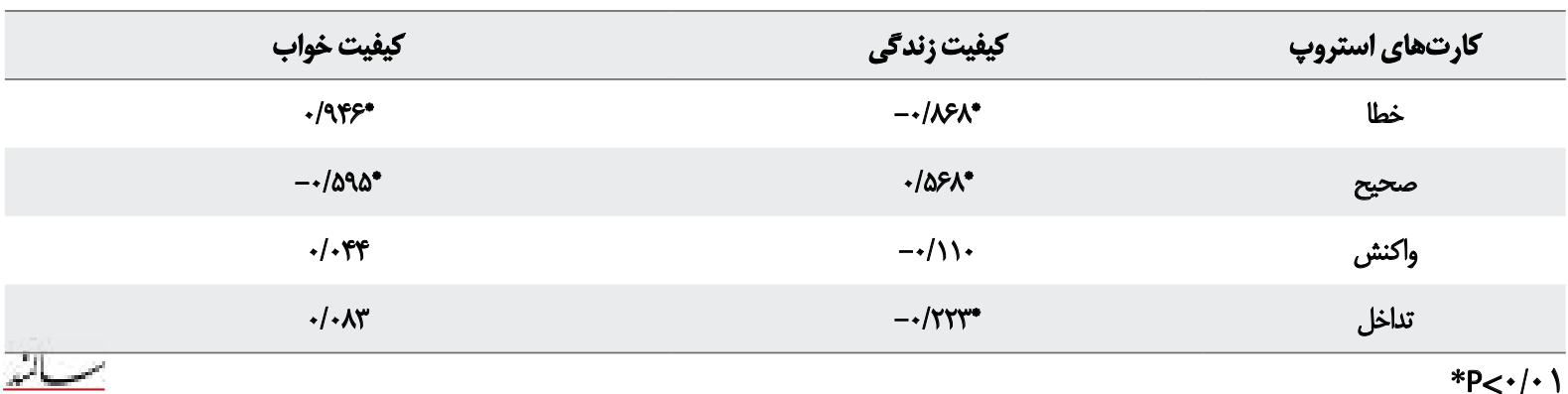

توصيفى متغيرهاي مطالعهشده در جدول شماره ا كزارش شده است.

همانطور كه در جدول شماره ا مشاهده مي شود ميانكين و

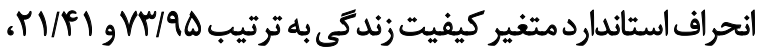

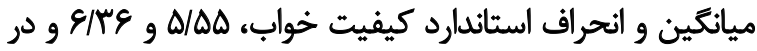

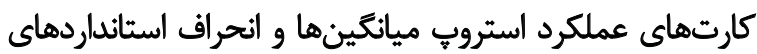
و r// ميزان رابطه كيفيت زندئى و كيفيت خواب با عملكرد استروض

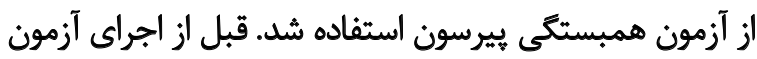

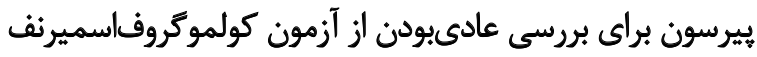

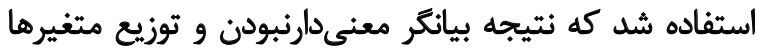

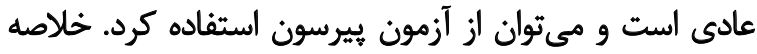

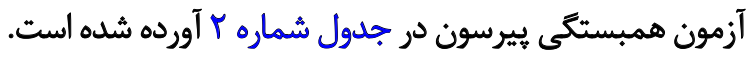

همانطور كه در جدول شماره r قابل مشاهده است، رابطه

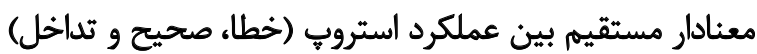

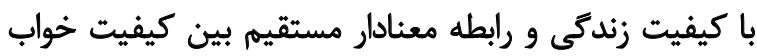

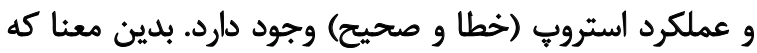

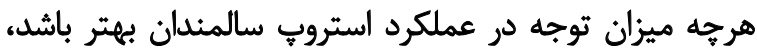

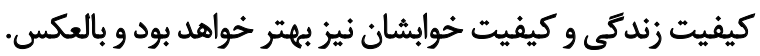

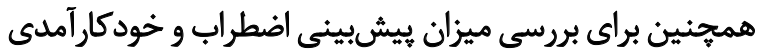

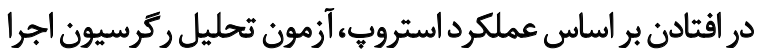

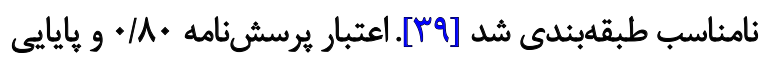

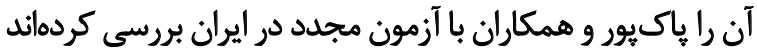

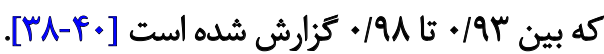

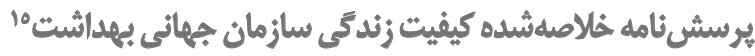
يرسشنامه خلاصهشده كيفيت زندكي سازمان جهاني

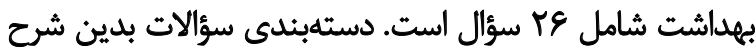

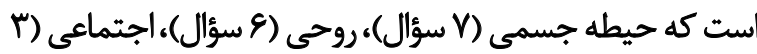

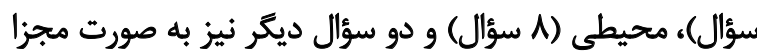

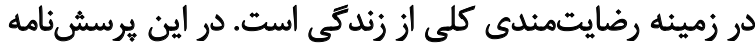

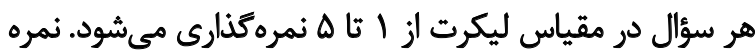

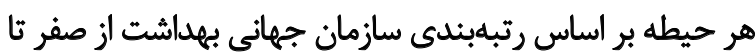

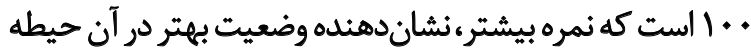

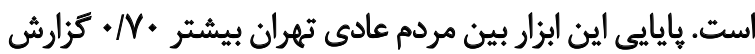

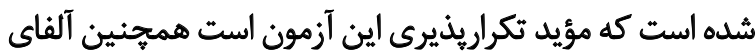

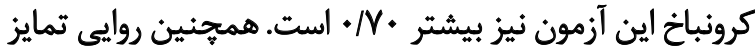

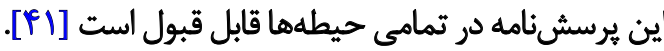

Latôt

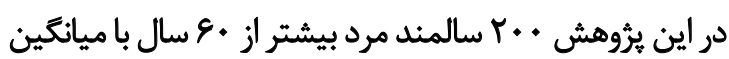

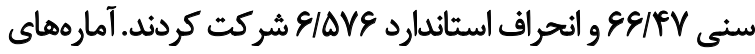

15. World Health Organization Quality of Life Questionnaire 
جدول r. نتايج ركرسيون براي بيشبينى كيفيت زندكى و كيفيت خواب براساس عملكرد توجه

\begin{tabular}{|c|c|c|c|c|c|c|c|}
\hline$P$ & $\mathbf{R}^{r}$ & $\mathrm{~T}$ & بثا & انحراف استاندارد & B & ملاكى & ييشبين \\
\hline $.1 . .1$ &.$/ 2 q$. & 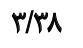 & $\cdot / r+1$ & $\cdot \mid \cdot m$ & .11 .8 & كيفيت زندكى & \\
\hline $.1 \%$ & .189. & P/AR &.$|r \Delta|$ & $.11 \cdot a$ & $\cdot / 4 \cdot \Delta$ & كيفيت خواب & \\
\hline
\end{tabular}

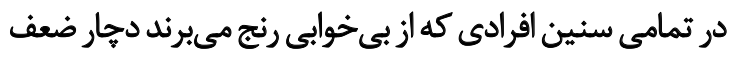

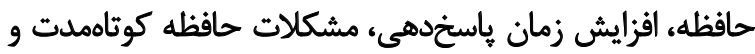

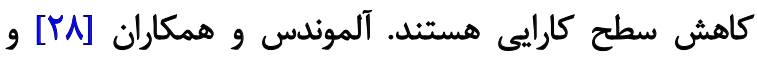

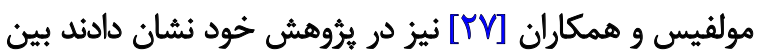

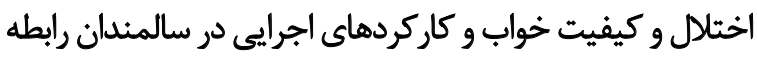

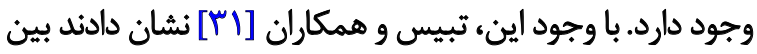

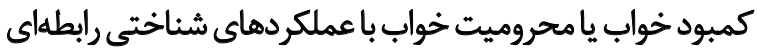

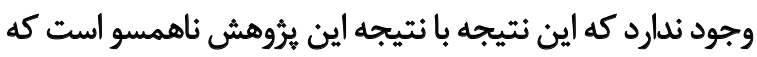

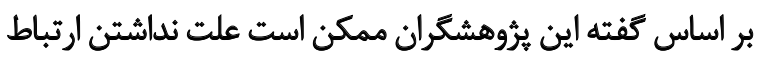

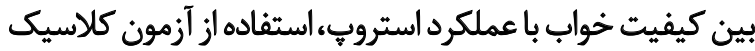

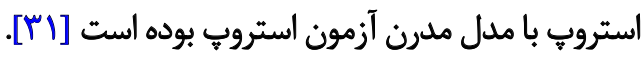

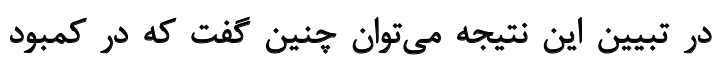

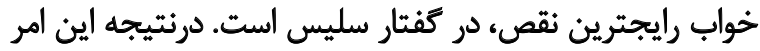

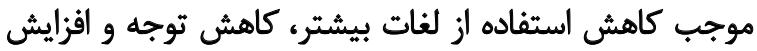

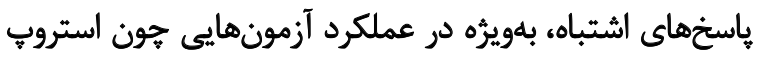

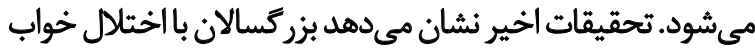
نسبت به كساني كه كيفيت خواب بهتردي

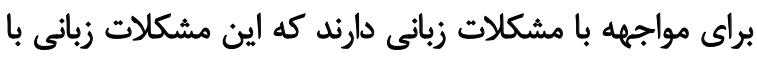

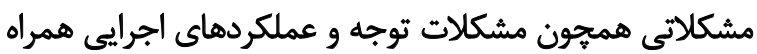

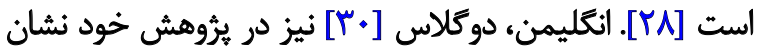

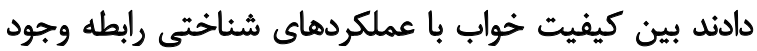

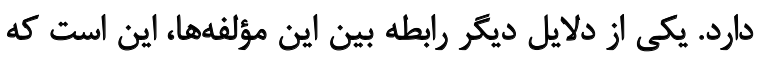

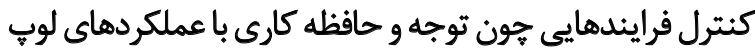

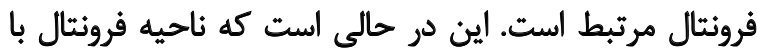

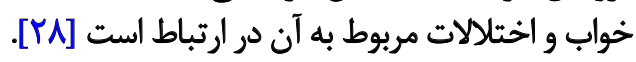

همجئنين نتيجه ديكر يروهش نشان داد بين عملكرد استروب

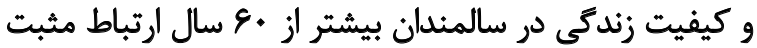

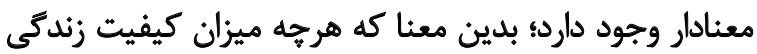

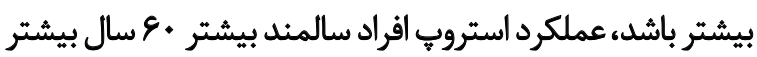

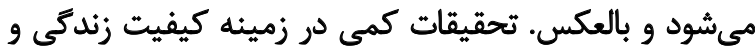

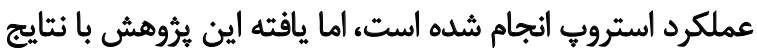

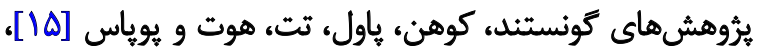

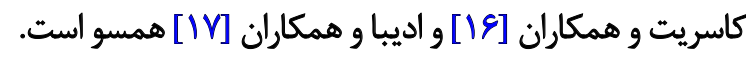
تحقيقات نشان مىدهد اضطراب، استرس، افسردكى و نقض

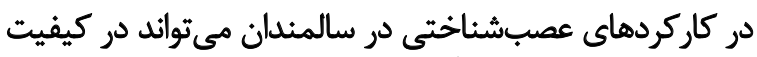

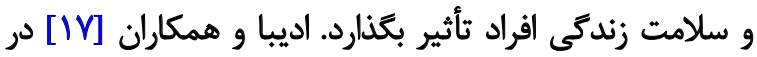

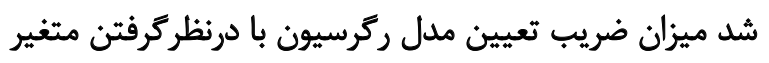

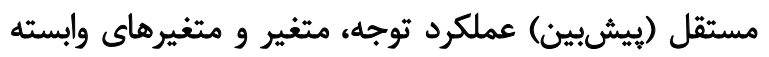

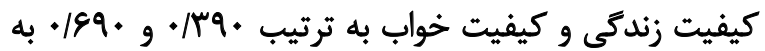

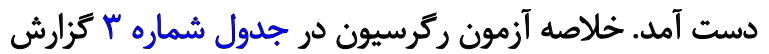
شده است.

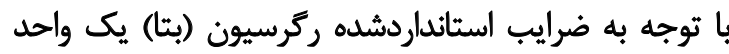

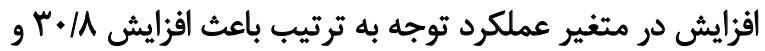

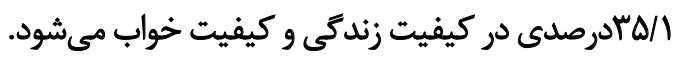

ث)

اين يُوهش با هدف بررسى عملكرد استروب با كيفيت خواب

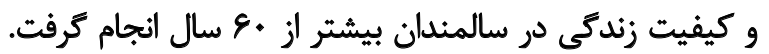

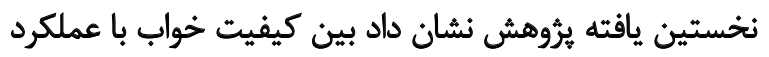

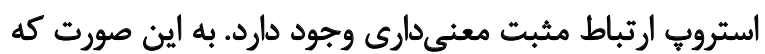

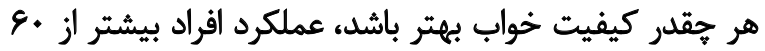

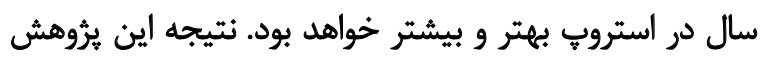

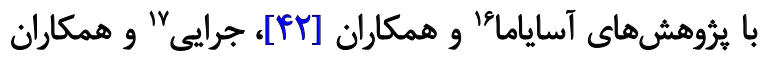

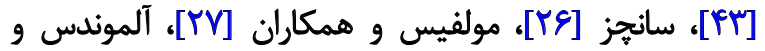

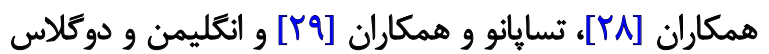

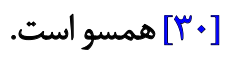

تزديك به يكسوم دوران زندكى انسان در خواب مي كذردي.

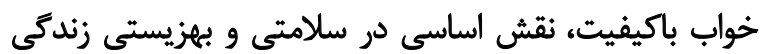

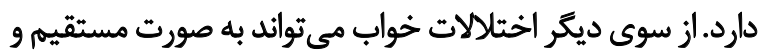

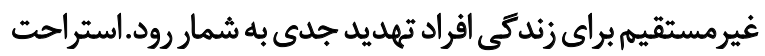

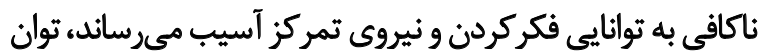

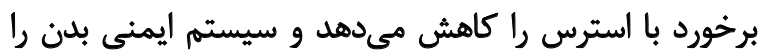

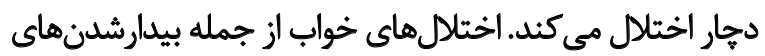

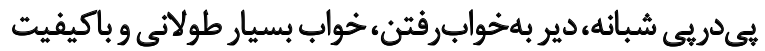

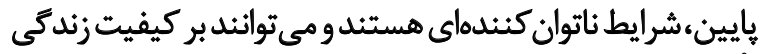

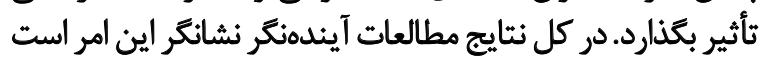

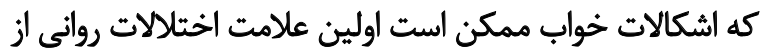

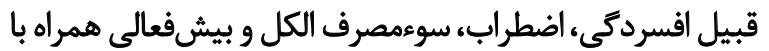

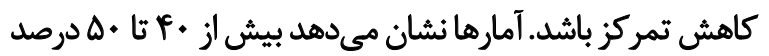
بزرَّالان بيشتر از •ه سال، بـ خوابى دارند.

16. Asayama

17. Jarraya 
غيرمستقيم ميزان كيفيت زندكى را افزايش داد و كيفيت خواب

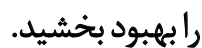

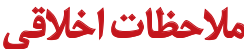

\section{يبيروى أز اصول الخلاث ئروهش}

به منظور رعايت اصول اخلاقى يُؤوهش يس از ازئتخاب

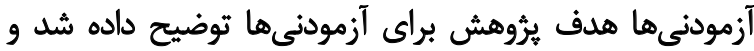

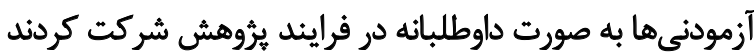

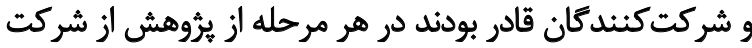

خود انصراف دهند.

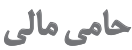

اين مقاله حامى مالى ندارد.

$$
\text { مشاركت نويسند أنان }
$$

مفهومسازي: همه نويسندكان؛ روش شناسى: همه نويسندكان؛

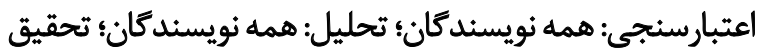

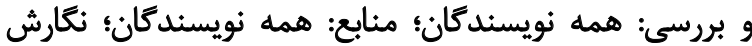

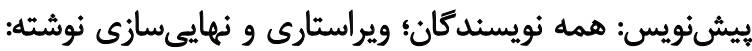

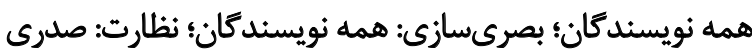

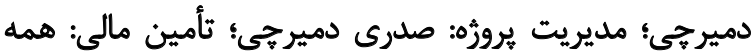

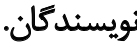

$$
\text { تقارض مثافع }
$$

بنا به اظهار نويسندكان، در اين مقاله هيجّكونه تعارض منافعى

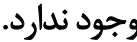

$$
\text { تشيكر و قبدرواني }
$$

بدين وسيله از همكارى مسئول محترم مركز رايحه اميد قدردانى مي وشود.

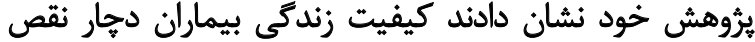

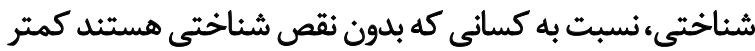

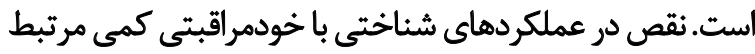

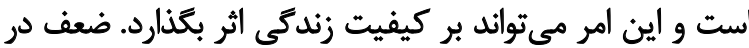

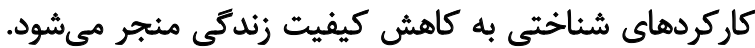

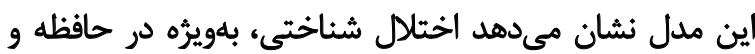

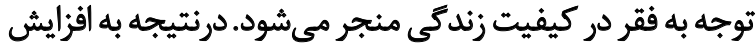

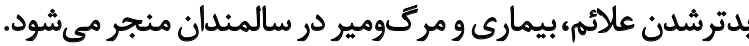

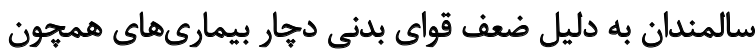

$$
\text { نار احتى قلبى، افسردكى و اضطراب مي قيوند. }
$$

در راستاى اين موضوع عملكردهاى شناختى افراد سالمند

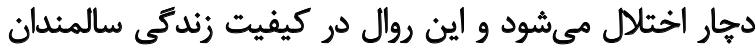

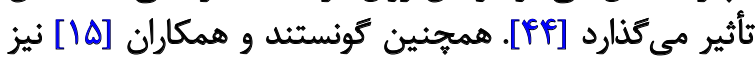

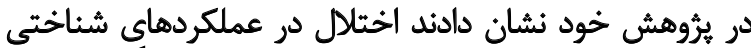

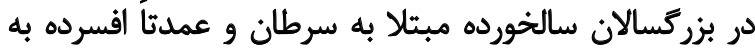

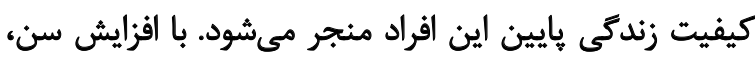

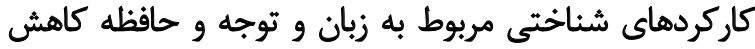

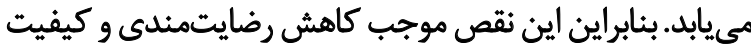

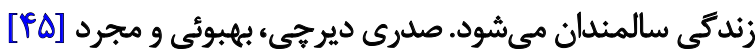

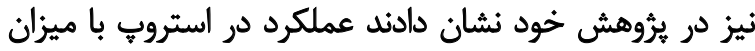

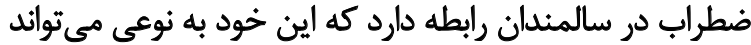
در كيفيت زندگى سالمندان مؤثر باشد.

يكى از محدوديتهاى اين يُوهش اين بود كه فقط روى إنى

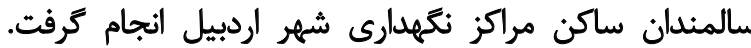

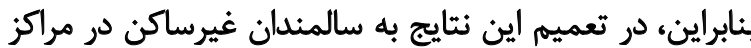

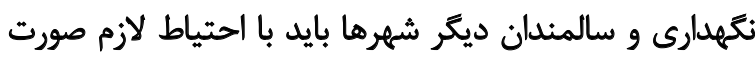

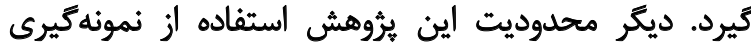

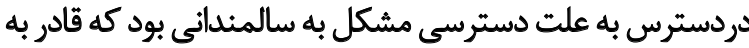

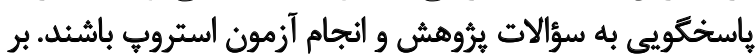

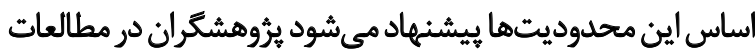

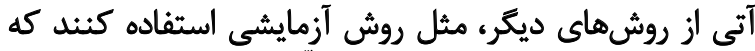

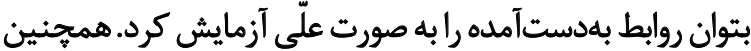

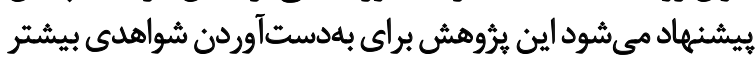

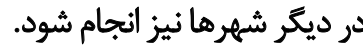

\section{تئيجهيرى نُهايى}

از آنجايى كه نتايج اين يُروهش آشكار كرد بين كيفيت خواب

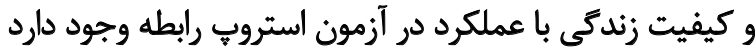

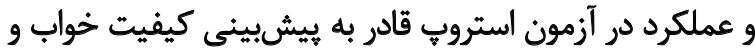

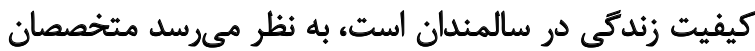

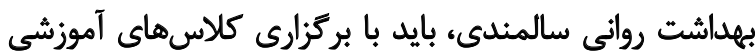

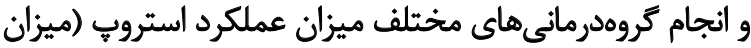
توجه) را در سالمندان بهبود بخشيد ثا بدين وسيله به طورئف 


\section{References}

[1] Payahoo L, Khaje-bishak Y, Pourghasem B, Asghari Jafarabadi $\mathrm{M}$, Mohammad-Bagher $\mathrm{K}$. [The survey of the relationship between quality of life of elderly with depression and physical activity in Tabriz, Iran (Persian)]. Scientific Journal of Rehabilitation Medicine. 2013; 2(2):39-46.

[2] Merzaei M, Ghafar khoei M. Elderly population in Iran based on accidents 1325-1385. 2006; 6:331

[3] Netz Y, Dwolatzky T, Zinker Y, Argov E, Agmon R. Aerobic fitness and multidomain cognitive function in advanced age. International Psychogeriatrics. 2011; 23(1):114-24. [DOI:10.1017/ S1041610210000797]

[4] Depp CA, Jeste DV. Definitions and predictors of successful aging: A comprehensive review of larger quantitative studies. The American Journal of Geriatric Psychiatry. 2006; 14(1):6-20. [DOI:10.1097/01.JGP.0000192501.03069.bc]

[5] Xavier FM, Ferraz M, Marc N, Escosteguy NU, Moriguchi EH. Elderly people s definition of quality of life. Brazilian Journal of Psychiatry. 2003; 25(1):31-9. [DOI:10.1590/S1516-44462003000100007]

[6] Bar-Haim Y, Lamy D, Pergamin L, Bakermans-Kranenburg MJ, Van Ijzendoorn $\mathrm{MH}$. Threat-related attentional bias in anxious and nonanxious individuals: A meta-analytic study. Psychological Bulletin. 2007; 133(1):1-24. [DOI:10.1037/0033-2909.133.1.1] [PMID]

[7] Williams JM, Mathews A, MacLeod C. The emotional Stroop task and psychopathology. Psychological Bulletin. 1996; 120(1):324. [DOI:10.1037/0033-2909.120.1.3] [PMID]

[8] Clark DA. Cognitive- behavioral therapy for OCD. New York City: Guilford Press; 2007.

[9] Ghanei Gheshlagh R, Farajzadeh M, Karami M, Ahani R, Sayehmiri K. [Investigating the relation between health-related quality of life and sleep quality in the Kurdish elderly of Saqqez (Persian)]. Nursing Practice Today. 2016; 3(3):116-26.

[10] Felce D, Perry J. Quality of life: Its definition and measurement. Research in Developmental Disabilities. 1995; 16(1):51-74. [DOI:10.1016/0891-4222(94)00028-8]

[11] Andrist L, Andrist LC, Nicholas P, Wolf K. A history of nursing ideas. Burlington, Massachusetts: Jones \& Bartlett Learning; 2006.

[12] King CR, Hinds PS. Quality of life: From nursing and patient perspectives. Burlington, Massachusetts: Jones \& Bartlett Publishers; 2011.

[13] Nikpour M, Abedian Z, Mokhber N, Ebrahimzadeh S, Khani S. [Comparison of quality of life in women after vaginal delivery and cesarean section (Persian)]. Journal of Babol University of Medical Sciences. 2011; 13(1):45-50.

[14] Dos Santos Tavares DM, Fernandes Bolina A, Aparecida Dias F, dos Santos Ferreira PC, Jose HaasV. Quality of life of elderly. Comparison between urban and rural areas. Invest. Educ. Enferm. 2014; 32(3):401-13. [DOI:10.17533/udea.iee.v32n3a05] [PMID]

[15] Gunstad J, Cohen RA, Paul RH, Tate DF, Hoth KF, Poppas A. Understanding reported cognitive dysfunction in older adults with cardiovascular disease. Neuropsychiatric Disease and Treatment. 2006; 2(2):213-8. [DOI:10.2147/nedt.2006.2.2.213]
[16] Gathright EC, Fulcher MJ, Dolansky MA, Gunstad J, Redle JD, Josephson R, et al. Cognitive function does not impact self-reported health-related quality of life in heart failure patients. The Journal of Cardiovascular Nursing. 2016; 31(5):405-11. [DOI:10.1097/ JCN.0000000000000277] [PMID] [PMCID]

[17] Ediebah DE, Reijneveld JC, Taphoorn MJ, Coens C, Zikos E, Aaronson NK, et al. Impact of neurocognitive deficits on patientproxy agreement regarding health-related quality of life in lowgrade glioma patients. Quality of Life Research. 2017; 26(4):86980. [DOI:10.1007/s11136-016-1426-z] [PMID] [PMCID]

[18] Taavoni S, Ekbatatni N, Kashaniyan M, Haghani M. Effect of Sedamin capsule on sleep disorder among menopausal women. Journal of Gorgan University of Medical Sciences. 2012; 14(1):40-4.

[19] Faraut B, Boudjeltia KZ, Vanhamme L, Kerkhofs M. Immune, inflammatory and cardiovascular consequences of sleep restriction and recovery. Sleep Medicine Reviews. 2014; 16(2):137-49. [DOI:10.1016/j.smrv.2011.05.001] [PMID]

[20] Kamphuis J, Meerlo P, Koolhaas JM, Lancel M. Poor sleep as a potential causal factor in aggression and violence. Sleep Medicine Reviews. 2012; 13(4):327-34. [DOI:10.1016/j.sleep.2011.12.006] [PMID]

[21] Grandner MA, Hale L, Moore M, Patel NP. Mortality associated with short sleep duration: The evidence, the possible mechanisms, and the future. Sleep Medicine Reviews. 2010; 14(3):191203. [DOI:10.1016/j.smrv.2009.07.006] [PMID] [PMCID]

[22] Aslani Y, Etemadifar S. Ali Akbari F, Heydari A. [Sleep disturbances in patients with heart failure obstructed in Hajjar Hospital, Shahrekord (Persian)]. University of Shahrekord Magazine. 2003; $9(1): 44-49$.

[23] Mousavi A, Khazaei H, Ghanbari Z, Abasi R. [Relationship between pain level and sleep quality in patients with headache (Persian)]. Journal of Research in Kermanshah University of Medical Sciences. 2014; 18(10):579-85.

[24] Ghafari F, Mohamadi S. [The frequency of sleepiness in women with high blood pressure (Persian)]. Scientific Journal of Hamedan Nursing and Midwifery Faculty. 2011; 9(1):5-13.

[25] Lima PF, Medeiros ALD, Araujo JF. Sleep-wake pattern of medical students: Early versus late class starting time. Brazilian Journal of Medical and Biological Research. 2002; 35(11):1373-7. [DOI:10.1590/S0100-879X2002001100016] [PMID]

[26] Herrero-Sánchez MD, García-Iñigo MD, Nuño-Beato-Redondo BS, Fernández-de-las-Peñas C, Alburquerque-Sendín F. Association between ongoing pain intensity, health-related quality of life, disability and quality of sleep in elderly people with total knee arthroplasty. Ciencia \& Saude Coletiva. 2014; 19(6):1881-8. [PMID]

[27] Molfese DL, Ivanenko A, Key AF, Roman A, Molfese VJ, $\mathrm{O}^{\prime}$ Brien LM, et al. A one-hour sleep restriction impacts brain processing in young children across tasks: Evidence from event-related potentials. Developmental Neuropsychology. 2013; 38(5):31736. [DOI:10.1080/87565641.2013.799169] [PMID] [PMCID]

[28] de Almondes KM, Costa MV, Malloy-Diniz LF, Diniz BS. The relationship between sleep complaints, depression, and executive functions on older adults. Frontiers in Psychology. 2016; 7:1-8. [DOI:10.3389/fpsyg.2016.01547] [PMID] [PMCID]

[29] Tsapanou A, Gu Y, O’Shea DM, Yannakoulia M, Kosmidis $\mathrm{MH}$, Dardiotis E, et al. Dataset on the associations between sleep quality/duration and cognitive performance in cognitively 
healthy older adults. Data in Brief. 2017; 14:720-3. [DOI:10.1016/j. dib.2017.08.028] [PMID] [PMCID]

[30] Engleman HM, Douglas NJ. Sleep - 4: Sleepiness, cognitive function, and quality of life in obstructive sleep apnoea/hypopnoea syndrome. Thorax. 2004; 59(7):618-22. [DOI:10.1136/ thx.2003.015867] [PMCID]

[31] Nebes RD, Buysse DJ, Halligan EM, Houck PR, Monk TH. Selfreported sleep quality predicts poor cognitive performance in healthy older adults. The Journals of Gerontology: Series B. 2009; 64B(2):180-7. [PMID] [PMCID]

[32] Hajari E, Bazargan A, Sarmaed Z. [Research methods in behavioral sciences (Persian)]. Tehran: Agah; 2012.

[33] Narimani M, Pouresmali A, Andalib KM, Aghajanei S. [A comparison of Stroop performance in students with learning disorder and normal students (Persian)]. Journal of Learning Disabilities. 2012; 2(1):138-58

[34] Gorfein DS, MacLeod CM. Inhibition in cognition. Washington: American Psychological Association; 2007. [DOI:10.1037/11587-001]

[35] Basharpour S. [The study of information processing speed, automated and controlled processing, and the effect of antidepressants on these three variables in depression disorder (Persian)] [MSc. Thesis]. Ardabil: University of Mohaghegh Ardabili; 2007.

[36] Aliloo Mm, Hamidi S, Shirvani A. [Comparison of executive function and sustained attention in students with obsessive-compulsive, high schizotypal and overlapping symptoms with the normal group (Persian)]. Journal of Research in Behavioral Sciences. 2011; 9(3):216-21.

[37] Knutson KL, Rathouz PJ, Yan LL, Liu K, Lauderdale DS. Stability of the Pittsburgh sleep. Sleep. 2006; 29(11):1503-6. [DOI:10.1093/sleep/29.11.1503] [PMID]

[38] Pakpour V, Zaman Zade V, Salimi S, Farsio A, Moghabeli Gh, Soheyli A. [Relationship between loneliness and quality of sleep in the elderly living in the elderly families of Tabriz (Persian)]. Journal of Urmia Nursing and Midwifery Faculty. 2016; 14(11):906-17.

[39] Fukuda H, Takahashi M, Miki K, Haratani T, Kurabayashi L, Hisanaga N, et al. Shift work-related problems in 16-h night shift nurses (1): Development of an automated data processing system for questionnaires, heart rate, physical activity and posture. Industrial Health. 1999; 37(2):219-27. [DOI:10.2486/indhealth.37.228] [PMID]

[40] Soleimany M, Masoodi R, Sadeghi T, Bahrami N, Ghorban M, Hassanpoor A. [General health and its relation with sleep quality in two groups of nurses working with the change in work at the educational centers of Iran University of Medical Sciences (IUMS) (Persian)]. Journal of Shahrekord University of Medical Sciences. 2008; 10(3):70-5.

[41] Nejat S, Montazeri A, Holakoei Naeini K, Mohammad K. [Standardization of the WHO Quality of Life Questionnaire (WHOQOL-BREF): Translation and psychometrics of the Iranian species (Persian)]. Journal of School of Public Health and Institute of Public Health Research. 2006; 4(4):1-12

[42] Asayama K, Yamadera H, Ito T, Suzuki H, Kudo Y, Endo S. Double blind study of melatonin effects on the sleep-wake rhythm, cognitive and non-cognitive functions in alzheimer type dementia. Journal of Nippon Medical School. 2003; 70(4):335-41. [DOI:10.1272/jnms.70.334] [PMID]
[43] Jarraya M, Jarraya S, Chtourou H, Souissi N, Chamari K. The effect of partial sleep deprivation on the reaction time and the attentional capacities of the handball goalkeeper. Biological Rhythm Research. 2013; 44(3):503-10.

[44] Amani O, Mazaheri MA, Nejati V, Shamsian BS. [Effect of cognitive rehabilitation on executive functions in adolescent survivors of leukemia: A randomized and controlled clinical trial (Persian)]. Archives of Rehabilitation. 2017; 18(1):73-82.

[45] Sadri Damirchi E, Behbuei S, Mojarrad A. [Role of performance in the stroop test in anticipation of anxiety and aggression in the elderly in Ardabil (Persian)]. Iranian Journal of Ageing. 2018; 13(1):38-49. 
This Page Intentionally Left Blank 